\title{
Dirac Cosmology and the Acceleration of the Contemporary Universe
}

\author{
Cheng-Gang Shao, Jianyong Shen, Bin Wand* \\ Department of Physics, Fudan University, \\ Shanghai 200433, People's Republic of China \\ Ru-Keng Sut \\ China Center of Advanced Science and Technology (World Laboratory) P.O. Box 8730, \\ Beijing 100080, People's Republic of China and \\ Department of Physics, Fudan University, \\ Shanghai 200433, People's Republic of China
}

\begin{abstract}
A model is suggested to unify the Einstein GR and Dirac Cosmology. There is one adjusted parameter $b_{2}$ in our model. After adjusting the parameter $b_{2}$ in the model by using the supernova data, we have calculated the gravitational constant $\bar{G}$ and the physical quantities of $a(t), q(t)$ and $\rho_{r}(t) / \rho_{b}(t)$ by using the present day quantities as the initial conditions and found that the equation of state parameter $w_{\theta}$ equals to -0.83 , the ratio of the density of the addition creation $\Omega_{\Lambda}=0.8$ and the ratio of the density of the matter including multiplication creation, radiation and normal matter $\Omega_{m}=0.2$ at present. The results are self-consistent and in good agreement with present knowledge in cosmology. These results suggest that the addition creation and multiplication creation in Dirac cosmology play the role of the dark energy and dark matter.

PACS numbers: 98.80.-k, 98.80.Cq
\end{abstract}

${ }^{*}$ Electronic address: wangb@fudan.edu.cn

${ }^{\dagger}$ Electronic address: rksu@fudan.ac.cn 


\section{INTRODUCTION}

According to the Dirac's arguments, the large dimensionless numbers provided by atomic physics and astronomy of our universe are connected with each other [1]. These numbers include: (i) the ratio of the electric to the gravitational force between an electron and a proton $a_{1}=e^{2} / G m_{p} m_{e} \sim$ $10^{39}$; (ii) the age of the universe expressed in terms of the atomic unit $a_{2}=\frac{m_{e} c^{3}}{e^{2} H} \sim 10^{39}$, where $H$ is the Hubble constant; (iii) the mass of the part of the universe which is receding from with a velocity $v<c / 2$, expressed in the units of the proton mass, say, $a_{3} \sim 10^{78}$. Dirac introduced a large number hypothesis

$$
a_{1} \cong a_{2} \cong a_{3}^{1 / 2}
$$

Based on this hypothesis, Dirac suggested a model of cosmology with a varying gravitational constant $G$ and an increase in the amount of matter in the universe.

Based on the large number hypothesis, a number of cosmological models with a varying gravitational constant have been proposed [2]-10]. However most of them met many difficulties. Noting that gravitational constant $G$ cannot vary in general relativity (GR), the first difficulty is that one must explain the contradiction between Einstein GR and Dirac Cosmology. Though much effort, for example, Milne two time scale hypothesis [6], Weyl's geometry [1] etc., has been devoted to reconcile the requirements of these two theories, it is still an open question to establish a theory which can unify the Dirac cosmology and Einstein GR.

The second difficulty comes from experiments. Almost all experiments at the scales of solar system and galaxies have not found the variation of $G$ [11]-[12]. A possible variation of $G$ has been investigated with no success through geophysical and astronomical observations. From experimental results one tends to believe that $G$ is a constant for local system with large scale.

The third difficulty belongs to the conservation of energy and momentum. Usually we use a perfect-fluid energy-momentum tensor to describe the matter of universe and it is conserved in the cosmic evolution. The addition creation, the new matter created uniformly in the whole space, and the multiplication creation, the new matter created in regions where old matters exist, must come from other mechanism as suggested by Dirac.

Many years ago a possible unified theory of Dirac cosmology and GR to overcome the above difficulties was suggested in [2]. The basic idea is as follows. Though one would expect a constant value of $G$ at the local system such as solar system, binary system and galaxies, it must be stressed that the cosmological observations still cannot put strong limits on the time variation of $G$ in the 
cosmological scale, especially at the late time of the universe evolution [10] 11]. Though Einstein GR has been proved to be correct by many experiments such as the excess perihelion precession of Mercury, gravitational redshift etc. at the scales of local system, it probably needs to be modified in the cosmological scale, especially if we want to use it to explain the recent observational result of the acceleration. Using the idea of Dirac cosmology with the variation of $G$ one could provide a possible way to modify the Einstein GR.

As is well known, the Lagrangian density of Einstein GR with a cosmological term is

$$
L_{E}=\frac{1}{16 \pi G} \sqrt{-g}(R-2 \Lambda)=\frac{1}{16 \pi G} \sqrt{-g} R(1-\alpha),
$$

where $\alpha=2 \Lambda / R$ is a dimensionless parameter. The cosmological constant $\Lambda$ can be generally explained as the background fluctuation of the cosmological vacuum, and $R$ is the 4-dimensional scalar curvature. In ordinary astrophysical problems of local systems, the magnitude of $R$ is about $8 \pi G \rho+4 \Lambda$. Since the vacuum energy density is much smaller compared to the density $\rho$ in local system, $\alpha$ is a very small quantity and can be neglected. But in cosmological problems, because $\rho$ is small and has the same order as that of the vacuum energy density, $\alpha$ cannot be neglected. In the limit of vacuum or the matter domination area, $\rho \rightarrow 0, R \rightarrow 4 \Lambda, \alpha$ can attain the magnitude $1 / 2$. This means that $\alpha$ plays an important role at cosmological scale. However, there is only a first order term of $\alpha$ in the equations of Einstein cosmology. Instead of the factor $1-\alpha$, we argue that in a perfect cosmological theory, the Lagrangian density of the gravitational field could contain higher-order terms of $\alpha$. We take the Lagrangian density as

$$
L_{E}=\frac{1}{16 \pi G} \sqrt{-g} R f(\alpha)
$$

where

$$
f(\alpha)=1-\alpha-b_{2} \alpha^{2}-\ldots
$$

The Einstein action becomes

$$
S=\frac{1}{16 \pi G} \int d^{4} x \sqrt{-g} R\left(1-\alpha-b_{2} \alpha^{2}-\ldots\right)+\int d^{4} x \sqrt{-g} L_{M} .
$$

Starting from Eq.(15), we can establish a Dirac cosmology with varying $G$ and matter creation. Obviously, this theory can unify GR and Dirac cosmology because in a local system with large scale, $\alpha \rightarrow 0$ and the theory reduces to Einstein GR. Only at cosmological scale, the terms $\alpha, \alpha^{2} \ldots$ become important and our theory reduces to Dirac cosmology.

Recently, many authors have considered the terms $R^{m}(m>1)$ and/or $R^{n}(n<0)$ on gravity 13]-[18]. But none of them has connected with Dirac cosmology and the large number hypothesis. 
In this paper, after introducing the higher order terms of $\alpha$, we will establish a unified theory of GR and Dirac cosmology and explain the acceleration of the contemporary universe, variation of $G$, cosmological supernova Type Ia data, and the creation of matter. We find that the addition creation of matter looks like the dark energy and the multiplication creation of matter like the dark matter.

The organization of this paper is as follows: we will present our model in Sec.II. In Sec.III, by using the present cosmological parameters as our initial conditions, we will calculate numerically the physical quantities of our universe and study the addition creation and multiplication creation. We will prove that our results are in good agreement with present knowledge in cosmology. Finally, a summary and conclusion will be given in Sec.IV.

\section{UNIFIED THEORY OF GENERAL RELATIVITY AND DIRAC COSMOLOGY}

Up to the second order of magnitude $O\left(\alpha^{2}\right)$, the action of our model is

$$
S=\frac{1}{16 \pi G} \int d^{4} x \sqrt{-g} R\left(1-\alpha-b_{2} \alpha^{2}\right)+\int d^{4} x \sqrt{-g} L_{M}
$$

where $b_{2}$ is a expansion parameter and we will adjust it from supernova data later. The field equation for the metric is then

$$
\left(1+b_{2} \alpha^{2}\right) R_{\mu \nu}-\frac{1}{2} R\left(1-\alpha-b_{2} \alpha^{2}\right) g_{\mu \nu}+b_{2} \alpha^{2} R^{2}\left(g_{\mu \nu} \nabla^{\sigma} \nabla_{\sigma}-\nabla_{\mu} \nabla_{\nu}\right) R^{-2}=8 \pi G T_{\mu \nu}^{M},
$$

where $T_{\mu \nu}^{M}$ is the energy-momentum tensor of matter and $\nabla_{\mu}(\mu=0,1,2,3)$ is the covariant derivative induced by the metric. When $b_{2} \neq 0$, the constant curvature vacuum solutions require

$$
\alpha=\frac{-1 \pm \sqrt{1+3 b_{2}}}{3 b_{2}} .
$$

We find the constant-curvature vacuum solutions are not Minkovski space, but rather de Sitter space $(+)$ or anti-de Sitter space $(-)$.

Eq.(77) can be rewritten as

$$
G_{\mu \nu}=8 \pi \bar{G}\left(T_{\mu \nu}^{M}+\theta_{\mu \nu}\right)
$$

where $G_{\mu \nu}$ is the Einstein tensor, and

$$
\begin{gathered}
\bar{G}=G /\left(1+b_{2} \alpha^{2}\right) \\
\theta_{\mu \nu}=-\frac{\Lambda}{8 \pi G} g_{\mu \nu}-\frac{1}{8 \pi G} \sqrt{\frac{4 b_{2} \Lambda^{2}(G-\bar{G})}{\bar{G}}} g_{\mu \nu}-\frac{1}{8 \pi}\left(g_{\mu \nu} \nabla^{\sigma} \nabla_{\sigma}-\nabla_{\mu} \nabla_{\nu}\right) \frac{1}{\bar{G}} .
\end{gathered}
$$


The physical meaning of $\bar{G}$ and $\theta_{\mu \nu}$ is obvious. We see from Eq.(9) that $\bar{G}$ plays the role of gravitational constant and $\theta_{\mu \nu}$ the creation of energy-momentum in the universe. They depend on the second order term $b_{2} \alpha^{2}$ of the expansion $f(\alpha)$. If $b_{2}=0, \bar{G}=G=$ const., $\theta_{\mu \nu}$ reduces to the ordinary vacuum energy cosmological term. Since $\bar{G}, \theta_{\mu \nu}$ depend on $R$ and then are functions of time $t$, this is just the character predicted by Dirac large number hypothesis. Futhermore, using Eq.(9) and the Bianchi identity, we get

$$
\nabla^{\nu} \theta_{\mu \nu}=-\left(T_{\mu \nu}^{M}+\theta_{\mu \nu}\right) \nabla^{\nu} \ln \bar{G}
$$

$\theta_{\mu \nu}$ is no longer conserved. To understand the physical meaning of Eq.(12), we rewrite it as following:

$$
\begin{aligned}
& \nabla^{\nu} \theta_{\mu \nu}^{(1)}=-T_{\mu \nu}^{M} \nabla^{\nu} \ln \bar{G} \\
& \nabla^{\nu} \theta_{\mu \nu}^{(2)}=-\theta_{\mu \nu} \nabla^{\nu} \ln \bar{G},
\end{aligned}
$$

where $\theta_{\mu \nu}^{(1)}+\theta_{\mu \nu}^{(2)}=\theta_{\mu \nu}$. It is clear that $\theta_{\mu \nu}^{(1)}$ is created around the normal matter, while the creation of $\theta_{\mu \nu}^{(2)}$ spreads over the whole universe. In the words of Dirac Cosmology, $\theta_{\mu \nu}^{(1)}$ is the multiplication creation having the basic character of dark matter and $\theta_{\mu \nu}^{(2)}$ is the addition creation having the basic character of dark energy. Both of them depend on the variation of $\bar{G}$. In Sec.III, the suggestions that addition and multiplication creations being dark energy and dark matter respectively will be elaborated numerically.

Employing flat Robertson-Walker metric

$$
d s^{2}=-d t^{2}+a^{2}(t) d \vec{x}^{2} \quad(k=0)
$$

and the perfect-fluid energy momentum tensor

$$
T_{\mu \nu}^{M}=\left(\rho_{M}+p_{M}\right) U_{\mu} U_{\nu}+p_{M} g_{\mu \nu}
$$

where $U^{\mu}$ is the 4 -velocity in fluid-rest frame, $\rho_{M}, p_{M}$ are the energy density and pressure respectively. Taking $p_{M}=w \rho_{M}$ as the equation of state, $w=0$ for matter, $w=1 / 3$ for radiation, we find that the field equations become

$$
\begin{gathered}
3 H^{2}=8 \pi \bar{G}\left(\rho_{M}+\rho_{\theta}\right)=8 \pi \bar{G} \rho_{M}+\frac{G}{\bar{G}} V(\bar{G})+3 H \frac{d \ln \bar{G}}{d t} \\
2 \dot{H}+3 H^{2}=-8 \pi \bar{G}\left(p_{M}+p_{\theta}\right)=-8 \pi \bar{G} p_{M}+\frac{G}{\bar{G}} V(\bar{G})-2 \frac{\dot{\bar{G}}}{\bar{G}}\left(\frac{\dot{\bar{G}}}{\bar{G}}-H\right)+\frac{\ddot{\bar{G}}}{\bar{G}},
\end{gathered}
$$




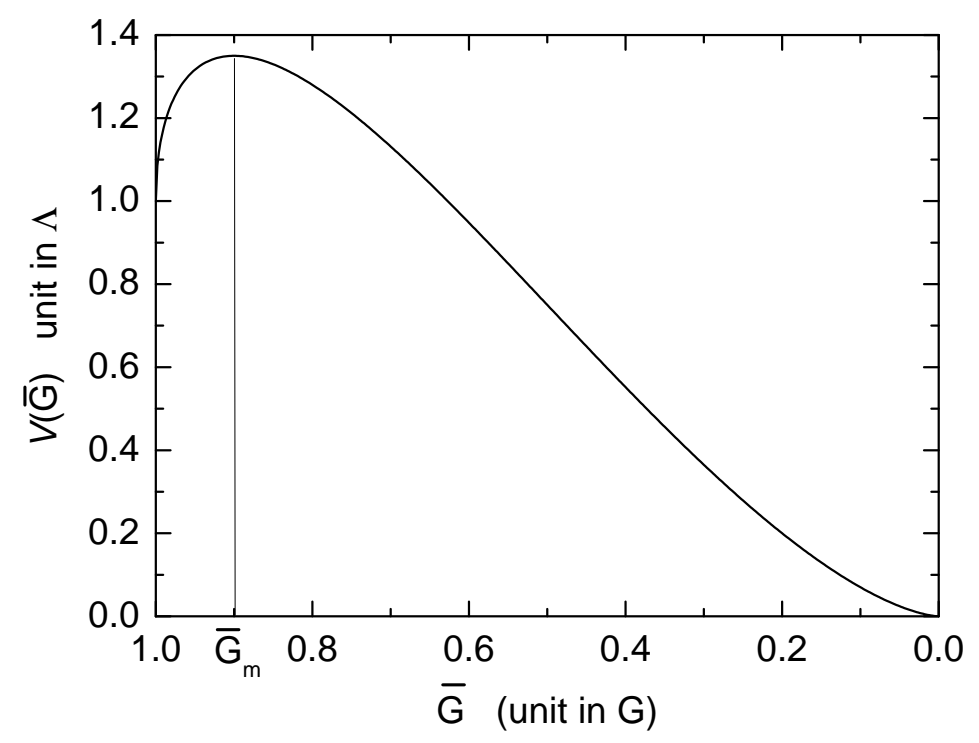

Figure 1: The potential $V(\bar{G})$ with $b_{2}=1$

where $H=\dot{a} / a, \rho_{\theta}=\theta_{00}, p_{\theta}=\theta_{i i} / g_{i i}(i=1,2,3)$ are the energy density and pressure of the created matter, respectively. Comparing to the kinetic term $\left(\frac{\dot{\bar{G}}}{G}\right)^{2}$,

$$
V(\bar{G})=\left(\frac{\bar{G}}{G}\right)^{2}\left\{\Lambda+\sqrt{4 b_{2} \Lambda^{2} \frac{G-\bar{G}}{\bar{G}}}\right\}
$$

which corresponds to a potential. Here we only consider the case of positive curvature scalar $R$. It is easy to show that $R$ is always positive in the evolution of the universe with matter and radiation. The kinetic term and the potential determine the evolution of $\bar{G}$ explicitly. $V(\bar{G})$ will reach its maximum at $\bar{G}_{m} \equiv G /\left[1+b_{2} /\left(1+\sqrt{1+3 b_{2}}\right)^{2}\right]$ as shown in Fig.(11).

We also define $\rho_{\theta^{(1)}}=\theta_{00}^{(1)}, \rho_{\theta^{(2)}}=\theta_{00}^{(2)}, p_{\theta^{(1)}}=\theta_{i i}^{(1)} / g_{i i}$ and $p_{\theta^{(2)}}=\theta_{i i}^{(2)} / g_{i i}(i=1,2,3)$ as the energy density and pressure of both of the multiplication and addition creation matter. The components of $\mu=0$ of Eq.(13) and Eq.(14) can be written as

$$
\begin{gathered}
\dot{\rho}_{\theta^{(1)}}+3 H\left(\rho_{\theta^{(1)}}+p_{\theta^{(1)}}\right)=-\rho_{M} \frac{\dot{\bar{G}}}{\bar{G}} \\
\dot{\rho}_{\theta^{(2)}}+3 H\left(\rho_{\theta^{(2)}}+p_{\theta^{(2)}}\right)=-\rho_{\theta} \frac{\dot{\bar{G}}}{\bar{G}}
\end{gathered}
$$

The other components of Eq.(13) and Eq.(14) $(\mu=1,2,3)$ give the identities about pressures $p_{\theta^{(1)}}$ and $p_{\theta^{(2)}}$ and no further information. Since the multiplication creation $\theta^{(1)}$ looks like dark matter, 
we set

$$
p_{\theta^{(1)}}=0 \quad p_{\theta^{(2)}}=p_{\theta}
$$

Eq.(17)-Eq.(22) and the equation of scalar curvature

$$
R=6\left[\frac{\ddot{a}}{a}+\left(\frac{\dot{a}}{a}\right)^{2}\right]=6\left(\dot{H}+2 H^{2}\right)
$$

given by the flat Robertson-Walker metric form a complete set. In principle, we can solve the set of equations to find the behavior of the cosmological evolution.

\section{THE NUMERICAL SOLUTIONS OF THE UNIVERSE WITH MATTER AND RA- DIATION}

We now study the behavior of our universe containing matter and radiation. To find the cosmological solution, we need the initial conditions of Eq.(17)-Eq.(22) in the early universe which, unfortunately, is little known now. Hence, we have done our numerical computation by using the present cosmological parameters as our initial conditions, which include the Hubble parameters constant $H_{0}=0.7 \times 100 \mathrm{~km} \cdot \mathrm{s}^{-1} M p c^{-1}$, the present deceleration $q_{0}=-0.5$, the cosmological constant $\Lambda=1$ (as the scale), the density of baryon $\Omega_{b}=0.05$, the ratio of the baryon-to-matter (including baryon and multiplication creation) $\Omega_{b} / \Omega_{m}=0.17$ and the ratio of baryon-to-photon $\eta=6.1 \times 10^{-10}[21]$. We will start from the present epoch and trace back the history of our universe.

Fig 2 shows the variation of $\bar{G}$ in the evolution. The present time is set as $t_{0}=0$. The three curves correspond to $b_{2}=0.66,2.5$ and 10 respectively. We see that the 'gravitational constant' $\bar{G}$ increases at first when the universe expands and then decreases at the late time. The ratio of the density of radiation to baryon floats up quickly and the scale factor $a(t) \rightarrow t^{1 / 2}$ as $t$ approaches the age of the universe, which are shown in Fig 3 and Fig 4 respectively. Therefore, the radiation dominates the early universe. And the age of the universe given by $a(t)=0$ is $1.02 G y r, 1.16 G y r$ and 1.34 Gyr for $b_{2}=0.66,2.5$ and 10 respectively. Since there is no mechanism of inflation in our model, it is easy to understand that our model can consider the universe back to the epoch of radiation domination. Fig [5] shows the evolution of the deceleration factor $q$. We see that the universe decelerates in the early era and gradually stops decelerating and starts accelerating. This is in a good agreement with what we understand the universe nowadays. Through the deceleration factor $q$, we notice that the scalar curvature $R$ is always positive.

Obviously, $b_{2}$ is the parameter in our model which needs to be adjusted. To determine this parameter, let us compare our model with the supernova data. Noting that the uncertainty of 


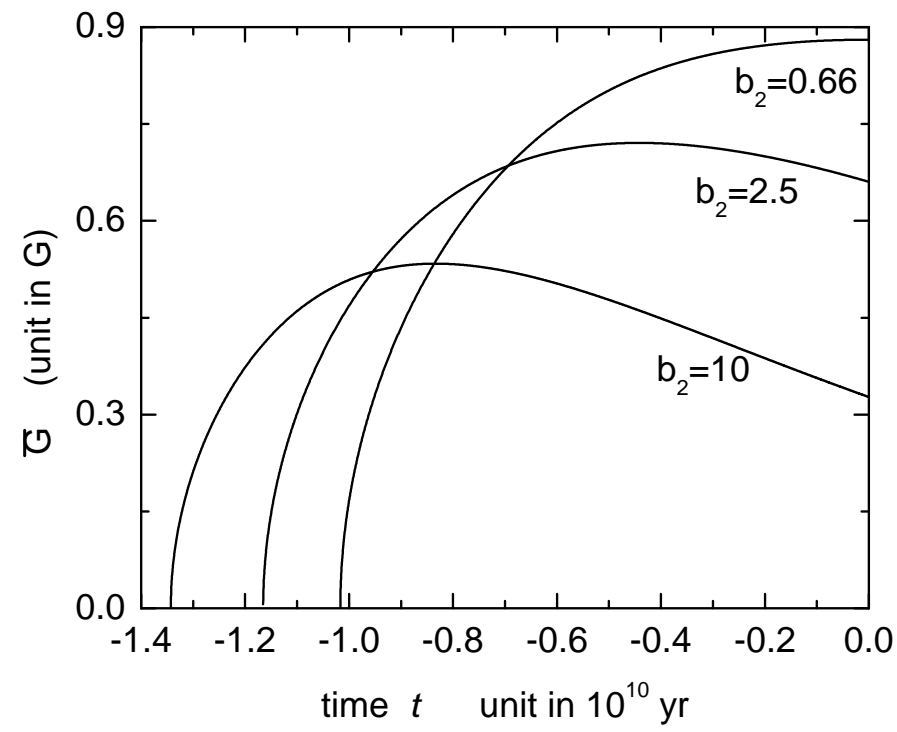

Figure 2: $\bar{G}$ versus time $t$. The present time is set as $t_{0}=0$. The three curves from the top to the bottom are for $b_{2}=0.66,2.5$ and 10 .

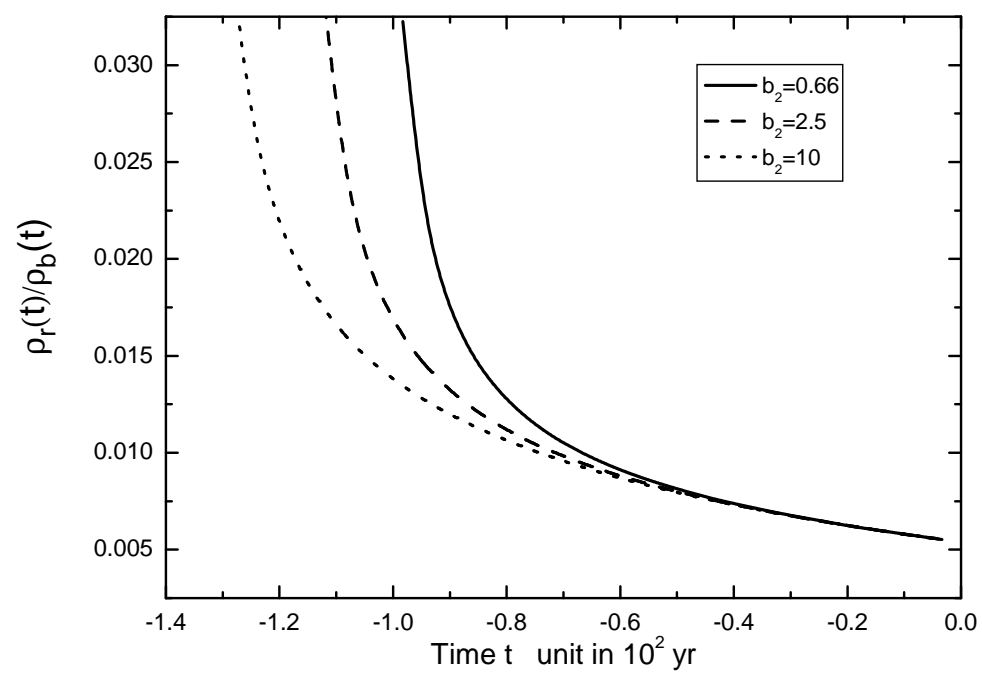

Figure 3: The ratio of the density of radiation-to-baryon $\rho_{r} / \rho_{b}$ versus time $t$.

the present value of the deceleration factor $q_{0}$ is still large, we will give a better estimation of the parameter $b_{2}$ and $q_{0}$ to fit the supernova data. Fig 6 presents the supernova data as a phase portrait of the universe [19] [20], where the universe was decelerating at high redshift and started accelerating when it was about two-third of the present size. Our model is favored by this set of data : $b_{2}=2.5$ and $q_{0}=-0.5$. Comparing Fig[6] and the curves given by Fig.2 of ref [20], we find 


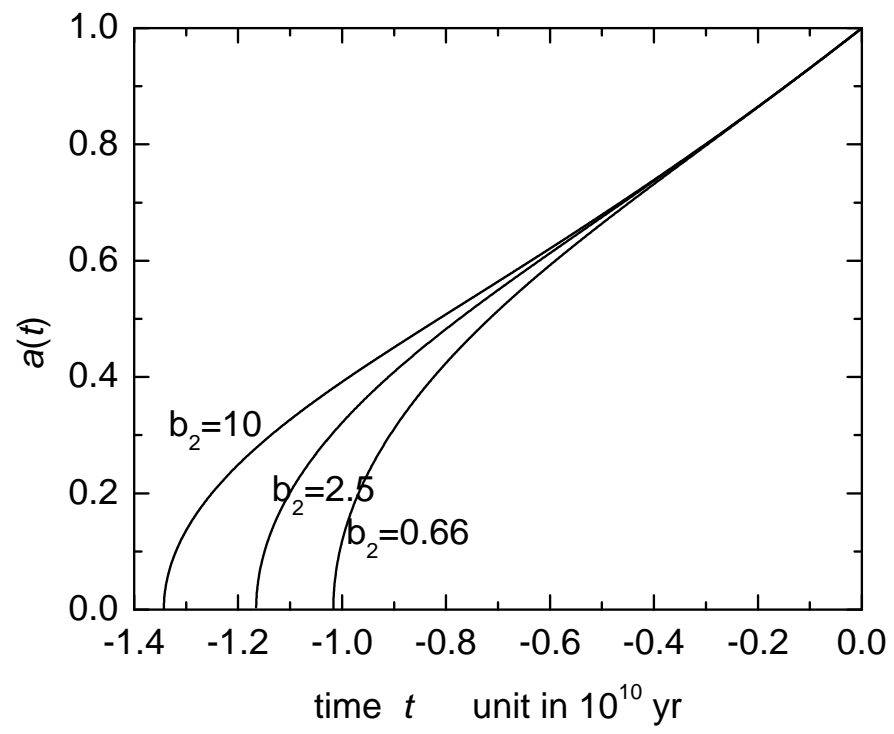

Figure 4: The scale factor $a(t)$ versus time $t$. The three curves from right to left correspond to $b_{2}=0.66,2.5$ and 10. All of them are asymptotic to $t^{1 / 2}$ as $t$ approaches the age of the universe.

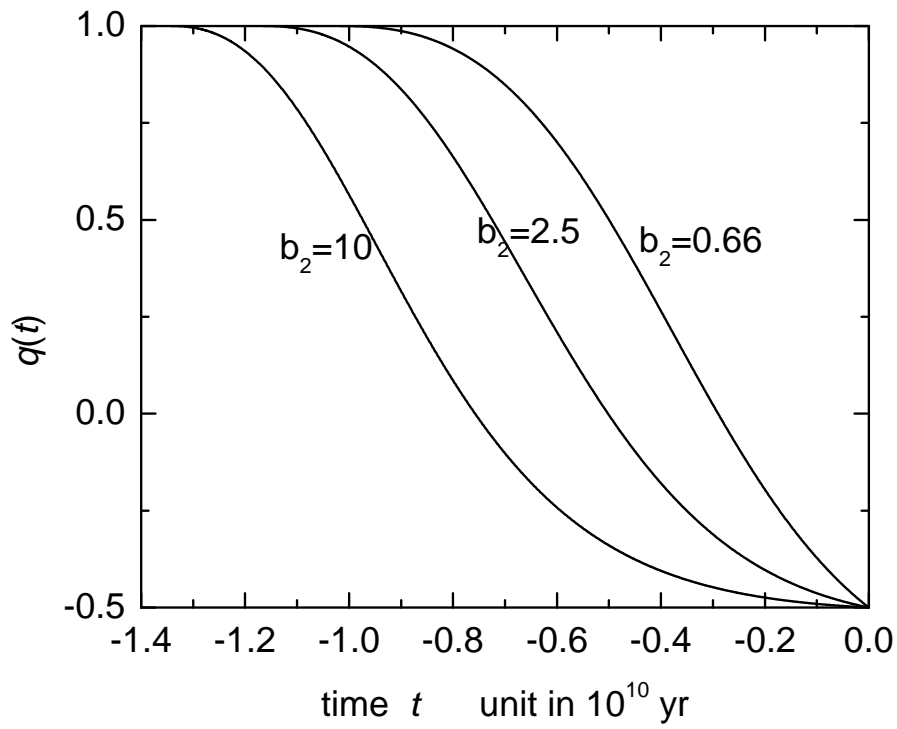

Figure 5: The evolution of the deceleration $q(t)$. The three curves from right to left correspond to $b_{2}=$ $0.66,2.5$ and 10 .

that the fit of our curve is better than that of the standard model.

Fig[7 shows the evolution of the pressure of the density of the addition creation matter with $b_{2}=2.5$. The pressure is highly positive in the beginning of the universe, falls quickly blow zero and has a negative value in the recent and present era. This negative pressure looks like the character 


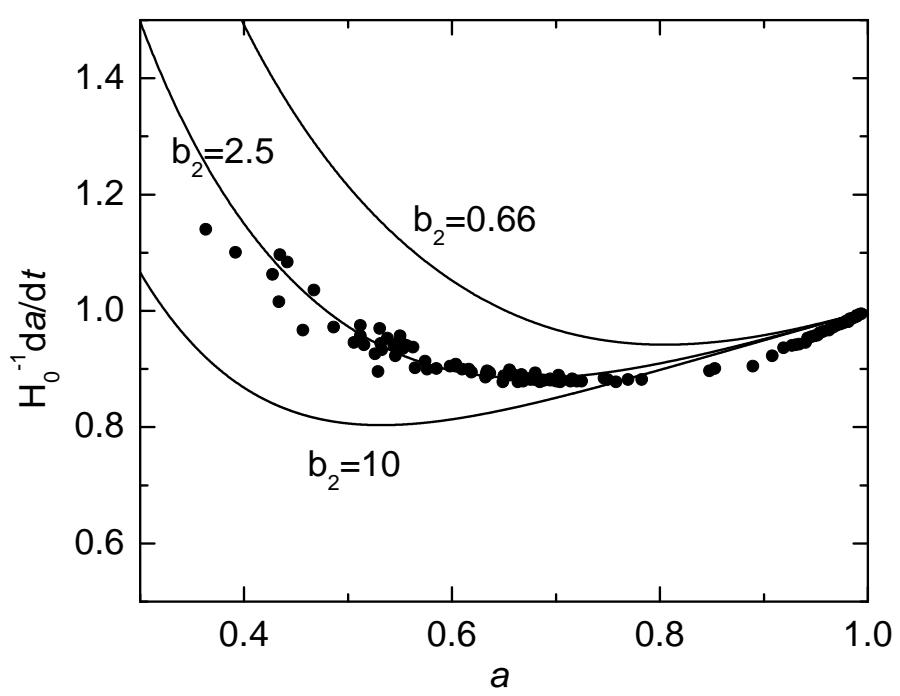

Figure 6: The "velocity" of the scale factor $\dot{a}$ versus the "position" $a$. The dots are the supernova data from 19 20]. The three curves from top to bottom correspond to $b_{2}=0.66,2.5$ and 10 .

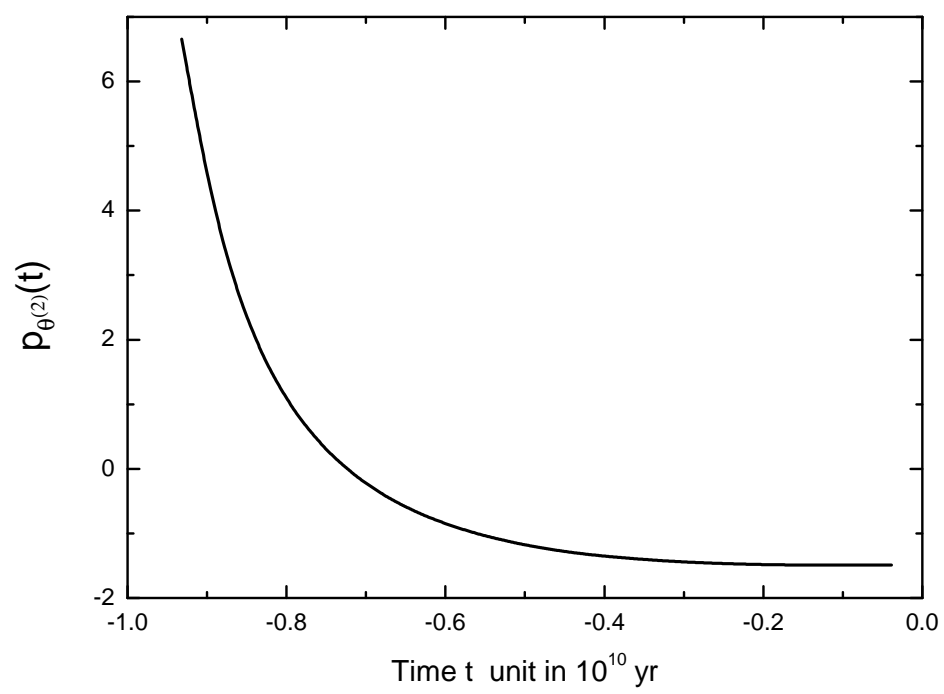

Figure 7: The evolution of the pressure of the addition creation $p_{\theta^{(2)}}$ at $b_{2}=2.5$.

of the dark energy. If we take $p_{\theta^{(2)}}=w_{\theta} \rho_{\theta^{(2)}}$ as the equation of state of addition creation, we show the parameter of the pressure-to-density of the addition creation $w_{\theta}$ versus time $t$ in Fig 8 and find $w_{\theta}(t=0)=-0.83$ which is much smaller than $-1 / 3$ and is consistent with the requirement of dark energy to explain the accelerating universe. To compare our results with other models, we 


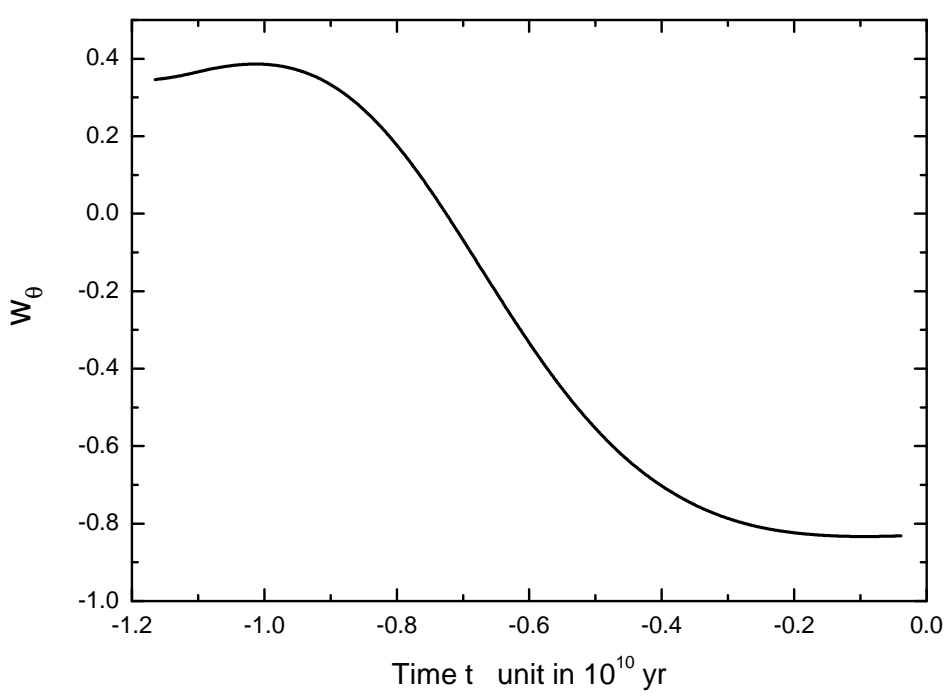

Figure 8: The evolution of the parameter of the pressure-to-density of the addition creation $w_{\theta}$ versus time $t$ at $b_{2}=2.5$.

have also calculated the ratio of the density of addition creation and find

$$
\frac{\rho_{\theta^{(2)}}}{\rho_{\theta^{(1)}}+\rho_{\theta^{(2)}}+\rho_{r}+\rho_{b}}=\Omega_{\Lambda}=0.8
$$

and that of the density of matter (multiplication creation and normal matter) in our model reads

$$
\frac{\rho_{\theta^{(1)}}+\rho_{r}+\rho_{b}}{\rho_{\theta^{(1)}}+\rho_{\theta^{(2)}}+\rho_{r}+\rho_{b}}=\Omega_{M}=0.2
$$

These results have the same magnitude as that dark energy and dark matter respectively. Hence, we suggest that the dark energy comes from the addition creation and the dark matter from the multiplication creation.

\section{SUMMARY AND DISCUSSION}

We have suggested a model to unify the Einstein GR and Dirac Cosmology. In local system, our theory reduces to GR, but in the cosmological scale, our theory refers to the Dirac cosmology. The variation of the gravitational constant comes from the scalar curvature. In the local system, the variation of the gravitational constant is negligible. This result is in good agreement with present experiments. But in the cosmological scale, the change of $\bar{G}$ is remarkable. The acceleration of the present universe is a proof of the decrease of the gravitational constant $\bar{G}$, because the decrease of $\bar{G}$ corresponds to an effective repulsion. 
After introducing the term $-b_{2} \alpha^{2}$ in our theory and fit the adjust parameter $b_{2}$ by supernova data, we have calculated the physical quantities of $\bar{G}(t), a(t), q(t), \rho_{r}(t) / \rho_{b}(t)$ by using the data of present epoch as the initial conditions. We have found that the results are self-consistent and in good agreement with present knowledge of cosmology.

According to Dirac large number hypothesis, matter will be created in the universe. We have calculated the matter comes from addition creation and that from multiplication. An interesting picture in our theory is that the addition creation, which spreads over the universe, looks like the dark energy and the multiplication creation, which clusters around the normal matter, like the dark matter. We have found that the pressure of addition creation has a big positive value initially and fall down quickly to become a negative value. It has a negative pressure region corresponding $0>t>-0.74 \times 10^{10} \mathrm{yr}$. The equation of state parameter $w_{\theta}=-0.83$ at present. This value is in agreement with present dark energy models. We have also calculated the ratio of the density of the addition creation and found $\Omega_{\Lambda}=0.8$. The same parameter but for multiplication creation, radiation and normal matter has also been computed, which reads $\Omega_{m}=0.2$. Both of them have the same magnitude of the observational value of the dark energy and matter. This result suggests that the dark energy and dark matter are just the addition creation and the multiplication creation in Dirac cosmology.

Finally, we would like to emphasize that this model cannot be extended to the big bang epoch. We have not add the terms with $R^{m}(m>1)$ (or $\left.\alpha^{-n}(n \geq 1)\right)$ in the Lagrangian density. Obviously, these terms are very important to the very early universe, especially in the inflationary epoch. Our model can only be used in the time evolution regions starting from the radiation dominated epoch to the present time.

\section{Acknowledgments}

This work was supported in part by NNSF of China, by the National Basic Research Program 2003CB716300 and the Foundation of Education of Ministry of China. B. Wang's work was also supported in part by Shanghai Education Commission.

[1] P.A.M. Dirac, Nature 139 (1937) 323, Pro. Roy. Soc. London A333 (1973) 403, Pro. Roy. Soc. London A365 (1979) 19 
[2] R.K. Su and S.W. Zhang, Proc. of the 3rd Grossmann Meeting on Gen. Rel. Ed. Hu Ning p1381, Sci. Press and North-Holland Pub Company (1983)

R.K. Su and S.W. Zhang, Acta. Math. Sci. 3 (1983) 321, Kexue Tongbao (Sci. Bullium of China) 27 (1982) 944

[3] G. Brans and R.H. Dicke, Phys Rev. 124 (1961) 925

[4] F. Hoyle and J.V. Narlikav, Proc. Roy. Soc. A227 (1964) 1

[5] H.W. Peng, gr-qc/0401105 gr-qc/0405002

[6] V. Canuto, et. al., Phys. Rev. Lett. 39 (1977) 429

[7] T. Damour and K. Nordtvedt, Jr., Phys. Rev. Lett. 70 (1993) 2217, Phys. Rev. D48 (1993) 3436

[8] T. Damour, F. Piazza and G. Veneziano, Phys. Rev. Lett. 89 (2002) 081601, Phys. Rev. D66 (2002) 046007

[9] K. Nordtvedt, Jr., gr-qc/0301024

[10] S. Carneiro and J.A.S. Lima, gr-qc/0405141, Gen. Rel. Grav. 26 (1994) 909

[11] J.P. Ozan, Rev. Mod. Phys. 75 (2003) 403

[12] J.G. Williams, S.G. Turyshev and D.H. Boggs, Phys. Rev. Lett. 93 (2004) 261101

[13] G. Magnano and L.M. Sokolowski, Phys. Rev. D50 (1994) 5039

[14] A. Dobado and A.L. Maroto, Phys. Rev. D52 (1995) 1895

[15] S. Capozziello, V.F. Cardone, S. Cardoni and A. Troisi, Phys. Lett. A326 (2004) 292

[16] S.M. Carroll, V. Duvvuri, M. Trodden and M.S. Turner, Phys. Rev. D70 (2004) 043528

[17] S. Nojiri and S.D. Odintsov, Phys. Rev. D68 (2003) 123512

[18] A.A. Starobinsky, Phys. Lett. B91 (1980) 99

[19] T. Padmanabhan, T.R. Choudhury, Mon. Not. Roy. Astron. Soc. 344 (2003) 828

[20] T.R. Choudhury and T. Padmanabhan, Astron. Astrophys. 429 (2005) 807

[21] C.L. Bennett, et al., ApJS 148 (2003) 1 